Arts

et Savoirs

\section{Arts et Savoirs}

12 | 2019

Révolution et évolution

\title{
La « marche du progrès »
}

Autour d'une métaphore

The March of Progress: about a metaphor"

\section{Aude Déruelle}

\section{CpenEdition}

\section{Journals}

Édition électronique

URL : http://journals.openedition.org/aes/2044

DOI : 10.4000/aes.2044

ISSN : 2258-093X

Éditeur

Laboratoire LISAA

Référence électronique

Aude Déruelle, "La « marche du progrès » », Arts et Savoirs [En ligne], 12 | 2019, mis en ligne le 24

février 2020, consulté le 02 mars 2020. URL : http://journals.openedition.org/aes/2044 ; DOI :

10.4000/aes.2044

Ce document a été généré automatiquement le 2 mars 2020.

Centre de recherche LISAA (Littératures SAvoirs et Arts) 


\title{
La « marche du progrès »
}

\author{
Autour d'une métaphore \\ The March of Progress: about a metaphor"
}

Aude Déruelle

1 L'expression «la marche du progrès " a été popularisée par une illustration de Zallinger en 1965 (March of Progress) qui a abondamment été reprise et détournée, mais elle est en réalité bien antérieure. En France, elle commence à s'imposer à partir de $1830^{1}$. Elle devient peu à peu une expression sinon lexicalisée, du moins si courante qu'il faut faire l'effort de la décomposer pour s'apercevoir qu'elle est étymologiquement redondante, puisqu'elle signifie en somme « la marche de la marche en avant $\rrbracket^{2}$ - et le succès de l'illustration est sans doute dû au fait qu'elle remotive le sens du terme « marche » en représentant la locomotion des hominidés (sa légende est d'ailleurs “The Road to Homo Sapiens"). Au-delà de cette valeur tautologique, qui n'est pas inintéressante pour autant, cette expression a des significations qu'il convient de mettre en perspective. En elle se noue une représentation du temps et de l'humanité au confluent de l'histoire et de la science. Sans revenir en détail sur la manière dont pensée de l'histoire et pensée du vivant ont pu s'entremêler, sous les auspices des événements révolutionnaires, à travers des représentations souvent imagées du temps et de la nature ${ }^{3}$, on peut rappeler que la pensée de la Révolution vue comme une catastrophe a coexisté avec l'idée que les états de la nature se sont succédé sous le coup de cataclysmes successifs. De même, l'émergence du modèle de la «marche du progrès » dans l'ordre des espèces coïncide avec l'idée d'une "marche du progrès » dans les sociétés humaines. L'expression "marche du progrès » désigne en effet plusieurs phénomènes, et regroupe même plusieurs conceptions du progrès, ce qui a sans doute contribué au succès de sa diffusion. Interroger cette métaphore de la "marche», les circonstances de son émergence et ses valeurs autour de 1830, permettra de revenir à nouveaux frais sur les conceptualisations et les représentations du progrès dans le premier XIX ${ }^{e}$ siècle.

2 En ce qui concerne l'évolution des sociétés humaines, le concept qui incarne le mieux la «marche du progrès » à la toute fin des années 1820 et qui contribue à forger cette image est sans aucun doute celui de « civilisation », tel qu'il a été configuré par Guizot 
dans ses cours à la Sorbonne, et sur lequel il convient de s'attarder. Voici la définition que l'historien en donne : « le premier fait qui soit compris dans le mot civilisation [...], c'est le fait de progrès, de développement; il réveille aussitôt l'idée d'un peuple qui marche, non pour changer de place, mais pour changer d'état; d'un peuple dont la condition s'étend et s'améliore. L'idée du progrès, du développement, me paraît être l'idée fondamentale contenue sous le mot de civilisation $»^{4}$.

Cette définition appelle deux remarques. Premièrement, le terme de "civilisation $»^{5}$ semble fonctionner comme un quasi synonyme de celui de "progrès ». Comme le rappelle Reizov, la notion était très à la mode durant la décennie : « Le mot civilisation est, pour ainsi dire, le mot d'ordre de notre siècle. $\|^{6} \mathrm{Ce}$ terme était déjà présent sous la plume des Idéologues, chez Condorcet, qui évoque régulièrement les différents "états » de la "civilisation" dans son Esquisse d'un tableau historique des progrès de l'esprit humain ${ }^{7}$. Deuxièmement, la définition développe l'image de la marche. Celle-ci est clairement métaphorique puisqu'elle ne renvoie pas, comme le souligne Guizot, à un déplacement spatial («non pour changer de place»), mais à une évolution sociale (" pour changer d'état»). Parmi d'autres auteurs, Staël parlait certes déjà de «la marche lente, mais continuelle de l'esprit humain dans la philosophie et de ses succès rapides mais interrompus dans les arts $»^{8}$. Mais la formule de Guizot ne vise pas seulement l'augmentation des connaissances humaines, elle renvoie bien à l'évolution même des sociétés. C'est « le peuple qui marche».

4 La civilisation, comme le détaille l'historien au début de son cours, se traduit en effet par un progrès dans deux domaines, moral et social. D’une part, « le développement de la vie individuelle, de la vie intérieure, le développement de l'homme lui-même, de ses facultés, de ses sentiments, de ses idées $»^{9}$. La civilisation touche à l'âme, et se révèle par les considérations religieuses, les ouvrages de l'esprit, de l'art, de la culture en un mot. D'autre part, la civilisation se caractérise par le progrès de l'activité sociale: amélioration des sociétés humaines, élaboration des lois, constitution des gouvernements, recherche du bien-être, progrès des industries. Or, dit Guizot, l'humanité a la "croyance instinctive » que « les deux éléments de la civilisation sont liés l'un à l'autre, et se produisent réciproquement ${ }^{10}$, quoiqu'ils ne soient pas mécaniquement liés dans leur avancée respective, l'un pouvant se produire indépendamment de l'autre - avant qu'ils ne finissent, un jour, par se rejoindre.

5 Reste à voir ce qu'apporte l'image de "marche » développée par Guizot. Les images permettent de donner une figuration sensible à la théorie du progrès, par nature fort abstraite ${ }^{11}$. Outre celle de la marche, on trouve ainsi celle du « cours» de l'histoire, qui assimile le déroulement du temps à l'écoulement continu d'un fleuve. Le "cours des choses ", expression fréquente, assigne une nécessité à l'évolution historique. Guizot développe cette image dans l'Histoire de la civilisation en France, parue peu après :

[...] ceci est un pas nouveau, et un pas immense, dans la route où nous marchons. Depuis que nous étudions les lois barbares, nous avançons de plus en plus vers le même résultat; la fusion des deux sociétés devient de plus en plus générale, profonde ; et dans cette fusion, à mesure qu'elle s'accomplit, l'élément romain, civil ou religieux, domine de plus en plus [...] Évidemment c'est en ce sens que coule le fleuve, vers ce but que tend le progrès des événements. ${ }^{12}$

6 Le progrès est semblable au « cours » d'un fleuve en ce qu'il coule dans un « sens ", a un «but », et que rien ne lui résiste. Ce qui signifie, in fine, que la civilisation constitue le cours général, le mouvement de l'histoire. Aussi, faire l'histoire de la civilisation, c'est faire, d'une certaine façon, l'histoire de l'histoire même - ce qui revient à fusionner la 
discipline et son objet. Dans les cours de Guizot, le déroulement de l'examen historique semble donc animé du mouvement même de l'histoire de l'humanité. Or l'image du fleuve est précédée de peu de celle de la marche, à laquelle elle est étroitement associée ("dans la route où nous marchons»). Au moins trois significations se dégagent de l'usage de cette métaphore.

7 Tout d'abord, avec cette image, la civilisation paraît un processus naturel ${ }^{13}$ (comme le fleuve). La métaphore estompe ainsi tous les heurts du progrès pour donner l'impression d'un mouvement régulier et continu. Ainsi que le dit Paule Petitier :

Guizot s'emploie à transformer la durée en espace uniforme, logique autant que chronologique, et à fondre la diversité des faits et la singularité des événements dans cette trame. Sous un certain angle, il hait l'événement, qui fait obstacle à la continuité du temps. ${ }^{14}$

8 C'est d'ailleurs le principe même du propos de Guizot, qui est de montrer en quoi tous les moments de l'évolution humaine se fondent au sein de cette marche lente mais sûre du progrès social, de la civilisation : « le despotisme, par exemple, et l'anarchie, s'ils ont contribué en quelque chose à la civilisation, s'ils lui ont fait faire un grand pas, eh bien ! jusqu'à un certain point, on les excuse, on leur pardonne leurs torts, leur mauvaise nature $»^{15}$. Dans l'ordre de l'histoire, un «mal» peut servir la cause du «bien» (le progrès, la civilisation) ${ }^{16}$.

9 En outre, la redondance de l'image ne fait que souligner son inéluctabilité : en somme, ce n'est pas tant l'humanité qui marche que le progrès qui s'accomplit. C'est la civilisation elle-même qui marche ${ }^{17}$. Plus loin, dans son cours sur la civilisation européenne, Guizot précise sa pensée : « ainsi coexistent les deux faits qui éclatent dans l'histoire de la civilisation, d'une part, ce qu'elle a de fatal, ce qui échappe à la science et à la volonté humaine, d'autre part, le rôle qu'y jouent la liberté et l'intelligence de l'homme, ce qu'il y met du sien parce qu'il le pense et le veut ainsi »18. Guizot réunit certes la valeur de la liberté humaine et une vision fataliste de l'histoire, mais on comprend que l'évolution des sociétés est mue par une détermination à laquelle l'humanité ne saurait se soustraire. Ainsi la redondance de la métaphore vise-t-elle à renforcer la nécessité de cette vision de l'histoire.

Enfin, et ce n'est pas le moindre des sens de cette métaphore, cette «marche » du peuple sur la « route » de la civilisation, qui en constitue une vision aussi naturelle que nécessaire, donne l'image d'un mouvement orienté vers une fin: on marche dans une direction - pour atteindre un but. Point de place au hasard, à la flânerie, à la déambulation, à l'esthétique du zigzag, dans cette image. C'est ainsi qu'est réintroduite subrepticement la question de la Providence :

La marche de la Providence n'est pas assujettie à d'étroites limites; elle ne s'inquiète pas de tirer aujourd'hui la conséquence du principe qu'elle a posé hier; elle la tirera dans des siècles, quand l'heure sera venue; et pour raisonner lentement, selon nous, sa logique n'est pas moins sûre. ${ }^{19}$

11 La «marche» du peuple sur la route du progrès est bel et bien l'expression de la «marche de la Providence»: le propos de Guizot permet ainsi de repenser la perfectibilité de l'humanité dans le cadre d'une vision transcendante et non immanente de l'histoire, vision transcendante qui toutefois est la plupart du temps sous-jacente au propos, mais bel et bien portée par la métaphore.

12 En ces dernières années de la Restauration, le cours de Guizot a remporté un prodigieux succès. Ce n'est peut-être pas tant la conception du progrès que Guizot développe à 
travers cette notion qui est novatrice que sa mise en scène à laquelle participe la métaphore de la marche. N'oublions pas d'ailleurs que le modèle du développement humain est la civilisation européenne - dans ses cours Guizot parle du «tour fâcheux de la civilisation musulmane $»^{20}$ - et plus précisément encore, la civilisation française, en laquelle se condense celle de l'Europe, et qui justifie que Guizot lui consacre un cours particulier : « Nous sommes bien placés pour nous adonner à cette recherche et étudier la civilisation européenne. Il ne faut flatter personne, pas même son pays ; cependant je crois qu'on peut dire sans flatterie que la France a été le centre, le foyer de la civilisation de l'Europe $»^{21}$. "Avec Guizot, tous les faits sociaux, politiques, religieux, culturels, s'ordonnent au sein d'une construction cohérente dont il suit l'ascension à travers les siècles. D'où la fascination de ses auditeurs pour cette mise en relief, productrice d'une nouvelle intelligibilité de l'histoire $»^{22}$. Certes, il y a dans ces pages la révélation d'un sens cohérent de l'histoire, qui est celui de son progrès. Mais le succès de ces cours repose aussi sur une série d'équations plus ou moins implicites (l'histoire = la civilisation $=$ le progrès $=$ l'Europe $=$ la France) qui place le public au cœur même de l'histoire, qu'il est invité à considérer, à analyser, et dont il constitue, pour ainsi dire, la force motrice.

13 Si la métaphore de la marche se déploie à la toute fin de la Restauration à travers le cours de Guizot pour désigner le progrès des sociétés humaines, cette expression n'est pas cantonnée au domaine de l'histoire, mais s'est également développée, de manière concomitante, à propos des sciences de la nature. Claude Blanckaert a fort bien montré comment les sciences naturelles ont conceptualisé, au début du siècle, «l'équation du temps et du perfectionnement biologique ». Il s'agit, dans cette pensée du progrès biologique, d'ordonner « en dynamique ascendante la série des êtres sur la flèche du temps $»^{23}$. Les modèles fondés sur la dégénération (Buffon) et la catastrophe (Cuvier), qui s'imposent au tournant du siècle, le cèdent à partir de 1830 à un modèle progressif d'une complexification du vivant avec le temps, les espèces disparues étant vues comme autant de premières étapes, plus simples et moins avancées, vers les espèces actuelles, qui représentent l'état définitif de la création. Il n'est d'ailleurs pas anodin que ce tournant coïncide avec les disparitions de Lamarck (1829) et de Cuvier (1832) ${ }^{24}$ puisqu'il ne s'agit plus en l'occurrence de transformisme ou de catastrophisme, et que ce progrès est aussi fixiste (les espèces n'évoluent pas, elles se succèdent).

En 1851, Marcel de Serres, promoteur de cette nouvelle vision du progrès du vivant, peut ainsi écrire : « Il devait, ce semble, en être ainsi, puisque, par la marche du progrès en raison directe de la complication de l'organisation, les oiseaux et les mammifères les plus perfectionnés ont apparu fort tard et ont précédé de peu la venue de l'homme en vue duquel ils avaient été créés ${ }^{25}$. Cette phrase montre bien la perspective finaliste dans laquelle cette "marche du progrès » est envisagée, l'avènement de l'homme étant perçu comme le sommet de la création. Ainsi, cette "paléontologie progressionniste procure à bon compte la garantie du sens $»^{26}$. Le succès de cette vision du progrès du vivant est sans doute, comme le rappelle Claude Blanckaert, dû au fait qu'elle permettait d'allier une vision providentialiste et la prise en compte d'une temporalité biologique. En une déclinaison de la vision de la genèse, l'homme est le couronnement de la « marche du progrès ». On voit à nouveau combien le terme de « marche » permet bien de faire jouer cette ambiguïté qui noue ensemble progrès et providentialisme: «Le vivant semble mû d'une force ascensionnelle sans être, à proprement parler, l'acteur de son développement. $»^{27}$ 

«marche du progrès », qui s'articulent autour d'un providentialisme souterrain, d'une progressivité orientée, d'une nécessité et d'une évidence de la progression. Et on comprend aussi que 1830 puisse avoir été la date pivot à partir de laquelle se diffuse cette métaphore - que ce soit pour les partisans ou les détracteurs du régime d'ailleurs. Les uns défendent l'avènement d'une monarchie raisonnable qui va accompagner la marche lente du progrès social - la Révolution de Juillet figurant une fin de l'histoire qui n'est pas pour autant une fin du progrès, vu alors comme une lente maturation, un perfectionnement moral et économique du pays ${ }^{28}:$ «la France désire l'amélioration, le progrès, mais une amélioration tranquille, un progrès régulier [...] que les amis du progrès de la civilisation et de la liberté n'aient aucune crainte [...] le perfectionnement social et moral est le résultat naturel de nos institutions ; il se développera librement et le gouvernement s'empressera de le seconder $»^{29}$. Les autres dénoncent les insuffisances du régime et réclament un progrès social et politique, ce qui explique que la Seconde République voit refleurir cette métaphore. On pense au projet de Chenavard pour le Panthéon, commandé par la Seconde République, La Palingénésie sociale, qui vise à peindre «la marche du genre humain dans son avenir à travers les épreuves et les alternatives de ruines et de renaissance $»^{30}$. Ou encore à la thèse exposée par Sue dans Les Mystères du peuple (1849-1857), qui ne cesse de marteler l'idée d'un "progrès constant de l'humanité »: «jamais l'humanité n'a reculé " $^{31}$, assène le héros en 1848. À la fin du roman, malgré l'échec de la révolution, l'auteur évoque à nouveau, par la bouche de son personnage, «la marche éternelle du progrès de l'humanité », puisqu'on a vu le peuple passer de l'état d'esclavage (des Romains puis des Francs) à l'exercice de la souveraineté grâce au double avènement républicain de 1792 et 1848: "Ah! lorsqu'on voit un tel progrès accompli à travers les siècles, comment douter du progrès que nous réserve l'avenir ? $\|^{32}$

image a certes eu ses détracteurs. Quinet critique par exemple la progressivité orientée d'une telle conception historienne : reprenant les mots de Guizot, il ironise sur le pardon que l'on accorde aux pires régimes car il faudrait à tout prix les faire entrer dans le déroulement logique d'une progression. La marche de la civilisation est le présupposé qui commande la lecture de l'histoire et l'interprétation de tout événement, aussi contraire puisse-t-il paraître au progrès :

On s'est dit: Prenons patience pendant la lente durée du moyen âge. Dans ce servage d'un peuple, voici l'aurore d'un grand jour qui luit sur nous. Les tentatives des communes avortent, les états généraux ne forment que des points clairsemés dans un espace trop souvent stérile; mais ces points épars marquent l'ébauche des constitutions parlementaires dans lesquelles se consomme la destinée de la France. 33

La logique même de successivité devient en somme un principe de causalité :

Ils disaient: Nous avons le régime parlementaire, qu'on l'appelle royauté ou république. Or cet état a été précédé d'une succession de rois absolus dans la vieille France; donc ce qui a précédé est cause de ce qui a suivi ; donc les princes absolus servent à préparer l'avènement des institutions libres; donc la formule générale de notre histoire est celle-ci: "En France, c'est le pouvoir absolu qui engendre la liberté !"34

Quinet démonte le présupposé historien qui nie les retours en arrière pour les subsumer dans la marche en avant du progrès. 
19 Mais la plasticité de cette métaphore de la marche, la dimension à la fois quasiment naturelle et objective du progrès qu'elle dégage, a permis à des représentations diverses du progrès de coexister et de s'épanouir - et, comme le souligne Claude Blanckaert dans le domaine biologique, cette représentation s'est imposée » malgré l'émergence des démonstrations de Darwin, car « elle s'identifie aux affects modernes » 35. Même lorsque la question de la transcendance est évacuée, l'image de la marche véhicule une foi dans le progrès qui s'est tout particulièrement déclinée dans la pensée positiviste ${ }^{36}$. Larousse, à l'article «Progrès " de son entreprise encyclopédique, peut écrire que le mot progrès désigne «la marche du genre humain vers sa perfection, vers son bonheur ", l'humanité allant « de la barbarie à la civilisation » (on retrouve en tout autre contexte les linéaments des réflexions de Guizot). Mêlant dans son article l'histoire et la science, il ajoute : " la foi à la loi du progrès est la vraie foi de notre âge ». Et de citer Le Monde marche d'Eugène Pelletan, qui fait l'apologie de la doctrine du progrès ${ }^{37}$.

20 Aux uns plait la linéarité d'une marche sans à-coup, sans heurts, qui interdit la pensée révolutionnaire en offrant une image rassurante du progrès: celui-ci semble alors s'accomplir de lui-même, notamment dans les domaines économique et industriel, et l'on peut tout au plus l'accompagner, le hâter ou le freiner, selon les termes souvent employés, mais en aucune manière l'empêcher. Les autres en revanche vont s'appuyer sur l'idée d'une avancée inéluctable du progrès pour minimiser les retours en arrière et les soubresauts politiques qui se succèdent dans le siècle, et galvaniser la marche en avant de l'humanité, à travers des luttes politiques et sociales.

La « marche du progrès » constitue ainsi une représentation qui touche à la mythologie tant elle frappe par sa persistance. Et il n'est pas sûr que la féroce ironie de Péguy, qui dénonce la vision d'un progrès continu en tournant l'image de la marche en dérision et en la dérivant obsessionnellement dans la métaphore de l'escalier, soit venue à bout de cette image :

Telle est la théorie du progrès. [...] C'est un escalier que l'on monte, et de qui l'on ne descend jamais, et où même l'on ne descend jamais, et de marche en marche toute acquisition de hauteur est acquise ; définitivement, sans perte ; finalement ; sans déperdition; et même sans frottement ; (car il faut qu'ils ignorent le frottement, et le tiennent égal à zéro) ; c'est un escalier bien fait ; tout marche qui vient après est forcément plus haute que toute marche qui vient avant ; on ne peut que monter ; on monte toujours ; on ne descend jamais, on ne peut pas descendre. ${ }^{38}$

\section{NOTES}

1. Selon une recherche effectuée sur la presse numérisée du site de la BnF, Retronews. L'expression a ses premières occurrences en 1831, dans les journaux Le Globe (racheté par Enfantin et les saint-simoniens à partir du mois d'août 1830) et Le Constitutionnel. Avant, le complément est au pluriel («la marche des progrès»), mais on trouve aussi l'expression «marcher de progrès en progrès ", ou bien encore, très souvent, les termes sont apposés : «la marche, le progrès ", en une forme de soulignement synonymique. 
2. Pour rappel, le substantif « progrès » vient du latin progressus (dérivé de progredior) qui signifie «marche en avant».

3. Sur ce sujet, on consultera l'ouvrage d'Olivier Ritz, Les Métaphores naturelles dans le débat sur la Révolution, Paris, Classiques Garnier, 2016, et notamment le chapitre «Les sciences révolutionnées?».

4. François Guizot, Histoire générale de la civilisation en Europe depuis la chute de l'Empire romain jusqu'à la Révolution française, Pichon et Didier, 1828, $1^{\text {re }}$ leçon, p. 15.

5. Cette notion a déjà fort bien été balisée par la critique. Citons pour mémoire l'analyse de Georges Gusdorf («Civilisation », Les Principes de la pensée au siècle des Lumières, Payot, 1971, p. 333-348), celle de Jean Starobinski (Le Remède dans le mal, Gallimard, 1989. Voir le premier chapitre, «Le mot civilisation »), qui reprend en partie les conclusions avancées par Benveniste, que voici: "De la barbarie originelle à la condition présente de l'homme en société, on découvrait une gradation universelle, un lent procès d'éducation et d'affinement, pour tout dire un progrès constant dans l'ordre de ce que la civilité, terme statique, ne suffisait plus à exprimer et qu'il fallait bien appeler la civilisation pour en définir le sens et la continuité. Ce n'était pas seulement une vue historique de la société; c'était aussi une interprétation optimiste et résolument non théologique de son évolution qui s'affirmait " (Émile Benveniste, "Civilisation : contribution à l'histoire du mot », Problèmes de linguistique générale [1966], Gallimard, « Tel », t. I, p. 340). On peut toutefois contester ce dernier point: les liens entre civilisation, progrès d'une part, et pensée providentialiste de l'autre sont bien plus ambigus. On renvoie à Karl Löwith, Histoire et salut. Les présupposés théologiques de la philosophie de l'histoire (1993), Paris, Gallimard, 2002, et notamment au quatrième chapitre, "Progrès contre Providence ».

6. La Revue encyclopédique [1825], cité par Boris Reizov, L'Historiographie romantique française, 1815-1830, Moscou, Éditions en langues étrangères, s.d. [1956], p. 284.

7. L'ouvrage, posthume [1794], a connu plusieurs rééditions dans les années 1820.

8. Germaine de Staël, De la littérature [1800], G. Gengembre et J. Goldzink éd., Flammarion, « GF », 1991, p. 65.

9. François Guizot, Histoire générale de la civilisation en Europe, $1^{\text {re }}$ leçon, p. 18.

10. Ibid., p. 23. Par son attention portée au " progrès de la vie individuelle, de la vie intérieure ", Guizot se distingue de Condorcet - quoiqu'en définitive il concentre son analyse sur le progrès social, ce qui peut paraître décevant au regard de son projet, et moins ambitieux. Surtout, son approche est plus historique que celle de son prédécesseur, dont l'esquisse est d'ordre philosophique. Il remet notamment en cause la vision rousseauiste de la société : le principe théorique d'un état naturel de l'homme en dehors de la société n'est aucunement heuristique. La mise en débat de l'héritage rousseauiste, nécessaire dans la perspective d'une réflexion sur les fondements philosophiques de la Révolution, est, comme le souligne Reizov, au cœur de la pensée libérale (op. cit., p. 296). C'est plus généralement l'héritage des penseurs politiques du XVIII ${ }^{\mathrm{e}}$ siècle qui est interrogé, notamment par les doctrinaires (Pierre Rosanvallon, Le Moment Guizot, Gallimard, «Bibliothèque des sciences humaines », 1985, p. 13-14 et p. 143 et sq.).

11. Ainsi a-t-on dit que «le progrès relève moins d'une histoire des concepts que d'une histoire des représentations » (Françoise Mélonio et Jacques Noiray, "Avant-propos », L'Idée de progrès, Romantisme $\mathrm{n}^{\circ} 108,2000$, p. 3-4).

12. François Guizot, Histoire de la civilisation en France, Pichon et Didier, t. I, 1829, p. 295.

13. Olivier Ritz a montré comment le recours aux «images naturelles» dit la nécessité du processus (op. cit., p. 85). Il ne faut pas oublier que les termes de «marche » et de «progrès » ont tous deux des acceptions astronomiques. Guizot écrit par exemple à sa femme : « mes regards se sont surtout dirigés vers l'ensemble des choses, sur la destinée de l'humanité, le cours, les lois, le but de son développement. C'est là surtout que l'intervention divine a éclaté à mes yeux, que j'ai reconnu clairement, irrésistiblement, la pensée et la volonté suprêmes. Je les trouve manifestes dans l'histoire du monde, d'une façon aussi certaine que dans la marche des astres; je vois Dieu 
dans les lois qui règlent le progrès du genre humain, aussi présent, aussi évident, bien plus évident selon moi, que dans celles qui président au lever et au coucher du soleil » (lettre citée par Reizov, op. cit., p. 282-283).

14. Paule Petitier, "Entre concept et hypotypose : l'histoire au XIX siècle ", Romantisme $\mathrm{n}^{\circ} 144$, 2009, p. 70. En ligne : https://www.cairn.info/revue-romantisme-2009-2-page-69.htm.

15. François Guizot, Histoire de la civilisation en Europe, op. cit., $1^{\text {re }}$ leçon, p. 9.

16. On retrouve ici à la fois une réappropriation de la problématique chrétienne du mal et un effet de la diffusion des idées hégéliennes, à laquelle Cousin a contribué durant les années 1820 .

17. «La Civilisation, reine gigantesque qui remplace le RoI, cette ancienne et terrible figure » (Honoré de Balzac, La Peau de chagrin, La Comédie humaine, Gallimard, «Bibliothèque de la Pléiade ", t. X, 1986, p. 108).

18. François Guizot, Histoire générale de la civilisation en Europe, op. cit., $11^{\mathrm{e}}$ leçon, p. 7.

19. François Guizot, Histoire de la civilisation en Europe, op. cit., $1^{\text {re }}$ leçon, p. 24 . En cela, Guizot se distingue fortement de Condorcet, qui refusait tout fondement théologique à la valeur de progrès. Sur ce «sens de la marche", voir aussi François Hincker, "L'effet d'utopie de la Révolution française », in M. Riot-Sarcey, (dir.), L’Utopie en questions, PUV, « La Philosophie hors de soi », 2001, p. 56-57.

20. Ibid., $3^{\mathrm{e}}$ leçon, p. 18.

21. Ibid., $1^{\text {re }}$ leçon, p. 4. Voit aussi cette assertion : «Il n'est presque aucune grande idée, aucun grand principe de civilisation qui, pour se répandre partout, n'ait passé d'abord par la France.» (ibid., p. 5)

22. Pierre Rosanvallon, introduction à son édition de l'Histoire de la civilisation en Europe, Hachette, «Pluriel», 1985, p. 31.

23. Claude Blanckaert, «Les fossiles de l'imaginaire. Temps de la nature et progrès organique (1800-1830)», Romantisme $\mathrm{n}^{\circ}$ 104, Penser avec l'histoire, p. 85. En ligne : https://www.persee.fr/ doc/roman_0048-8593_1999_num104_3410.

24. On pourra lire les discours prononcés à ces deux occasions par Geoffroy Saint-Hilaire et réunis dans ses Fragments biographiques (1838).

25. Marcel de Serres, Du perfectionnement graduel des êtres organisés, Bordeaux, T. Lafargue, 1851, p. 348.

26. Claude Blanckaert, art. cit., p. 93.

27. Ibid.

28. Pour une représentation de cette fin de l'histoire, voir la Salle 1830 des Galeries historiques de Versailles.

29. François Guizot, «Exposé de la situation du royaume présenté aux Chambres le 13 septembre 1830 ", repris dans les Mémoires pour servir à l'histoire de mon temps, Michel Lévy frères, t. II, p. 384.

30. «[...] cette lecture de la nature devait appuyer, vers 1840 , le nouvel idéal du progrès social » (Claude Blanckaert, art. cit., p. 96).

31. Eugène Sue, Les Mystères du peuple, Lachatre, Administration de Librairie, t. I, 1849, p. 135.

32. Ibid., t. XVI, p. 394. Sur la représentation de l'histoire dans ce roman, voir Aude Déruelle, «Historique et politique dans Les Mystères du peuple d'Eugène Sue », in P. Petitier et G. Séginger (dir.), Les Formes du temps. Rythme, histoire, temporalité, Strasbourg, Presses universitaires de Strasbourg, 2007, p. 81-94.

33. Edgar Quinet, Philosophie de l'histoire de France [1855], Paris, Pagnerre, 1857, p. 360.

34. Ibid., p. 361.

35. Claude Blanckaert, art. cit., p.99. Il rappelle ainsi que l'édition française de L'Origine des espèces a pour sous-titre « ou des lois du progrès chez les êtres organisés ».

36. Durant la décennie de 1830, dans ses Cours de philosophie positive, Comte noue ensemble, en une pensée systématique de la connaissance humaine, sciences et philosophie sociale, afin de dessiner la "marche progressive de l'esprit humain " (Bachelier, t. I, 1830, p. 2). Il développe 
dans le quatrième tome consacré à la philosophie sociale sa pensée du progrès, où il s'oppose à l'idée que l'avenir est borné par l'«avènement du christianisme au-delà duquel l'humanité ne saurait faire un pas »: «la condition de continuité constitue un élément indispensable de la notion définitive du progrès de l'humanité ", "la marche générale de ce développement graduel » (Bachelier, t. IV, 1839, p. 233-234). Mais ce progrès a une fin. Voir notamment, de Laurent Clauzade, "Auguste Comte et la naturalisation de l'esprit ", Methodos. Savoirs et textes $\left(2002, n^{\circ} 2\right)$, qui rappelle la position fixiste de Comte et le fait que le progrès est nécessairement un " processus fini qui doit s'achever avec l'état positif » : " La naturalisation du développement de l'esprit contribue donc à présenter le progrès comme un processus téléologique continu et déterminé, dont le principe est lui-même contenu dans l'organisation humaine. " En ligne: https://journals.openedition.org/methodos/61.

37. Eugène Pelletan, Lettres à Lamartine. Le Monde marche, Paris, Pagnerre, 1857.

38. Charles Péguy, Clio [1917], Paris, Gallimard, 1932, p. 49.

\section{RÉSUMÉS}

La métaphore de "la marche du progrès " commence à s'imposer en France à partir de 1830 . Cette expression a des significations que l'article met en perspective: en elle se noue une représentation du temps et de l'humanité au confluent de l'histoire et de la science. L'émergence du modèle de la "marche du progrès » dans l'ordre des espèces coïncide avec l'idée d'une «marche du progrès » dans les sociétés humaines. Bien plus, cette métaphore regroupe plusieurs conceptions du progrès, ce qui a contribué à sa diffusion. L'image construit une représentation naturelle du progrès, sa redondance soulignant son inéluctabilité. Elle élabore également une représentation $\mathrm{du}$ progrès orientée vers une fin, ce qui laisse place à un nouveau providentialisme, qu'il soit historique ou scientifique.

In France, the metaphor of "the march of progress" spreads from 1830. This image has different meanings that this article puts into perspective: it develops a representation of time and humanity at the confluence of history and science, as the emergence of a "march of progress" in natural species coincides with the idea of a "march of progress" in human societies. Moreover, this metaphor brings together several conceptions of progress, which has contributed to its dissemination. The image builds a natural representation of progress, the redundancy underlining its inevitability. It also develops a representation of progress towards an end, leading to a new providentialism in science and history.

\section{INDEX}

Keywords : progress, march, civilization, species, history

Mots-clés : progrès, civilisation, marche, espèces, histoire 
AUTEUR

AUDE DÉRUELLE

Université d'Orléans, POLEN 